\title{
Equations of Motion in the Theory of Relativistic Vector Fields
}

\author{
Sergey G. Fedosin \\ PO box 614088, Sviazeva str. 22-79, Perm, Perm Krai, Russia \\ E-mail: fedosin@hotmail.com
}

Keywords: vector field; equation of motion; field equations.

\begin{abstract}
Within the framework of the theory of relativistic vector fields, the covariant expressions are presented for the equations of motion of the matter and the field. These expressions can be written either in terms of the field tensors, that is, the fields' strengths and solenoidal vectors, or in terms the four-potentials, that is, the fields' scalar and vector potentials. This state of things is due to the fact that the Lagrange function initially implied the complementarity of description in terms of the strengths and the field potentials. It is shown that the equation for the fields, obtained by taking the covariant derivative in the equation for the metric, has a deeper meaning than the ordinary equation of motion of the matter, found with the help of the principle of least action. In particular, the abovementioned equation for the fields leads to the generalized Poynting theorem, and after integration over the volume it allows us to introduce for consideration the integral vector as a measure of the energy and the fields' energy fluxes, associated with a system of particles and fields.
\end{abstract}

\section{Introduction}

One of the most effective ways of obtaining the equations of motion is to apply the principle of least action [1-3]. In this case, as a rule, either the motion of charged particles in an electromagnetic field or the motion of particles in a potential field or in a scalar pressure field are described. As for the gravitational field, it usually appears as a tensor field within the framework of the general theory of relativity, including the use of modified versions of this theory to describe the dynamic properties of matter [4-6].

The concept of the electromagnetic field, which is a vector field, is successfully used in science and technology for a comprehensive description of electromagnetic phenomena. It is also possible to represent all the fields acting in macroscopic systems as the corresponding vector fields [7-9]. This allows us to apply the well-developed mathematical apparatus of the theory of relativistic vector fields and to describe with its help both the equation of the matter's motion under the action of the fields and the propagation of the fields during the matter's motion.

The main purpose of this paper is to present the equations of motion of the matter and field in a covariant form, which is suitable for use, in particular, in the curved spacetime and in any reference frames. We will systematize the results presented in the previous papers and will supplement them with regard to the equations for the fields' scalar and vector potentials.

Among a great number of vector fields, only the four fields will be considered, including the electromagnetic field, gravitation within the covariant theory of gravitation [10], the acceleration field and the vector pressure field [11]. Other macroscopic vector fields can be easily added to the presented equations, as has already been done for the dissipation field [12] and the fields of strong and weak interactions [13].

In our calculations, we will use everywhere the metric signature of the form $(+,-,-,-)$.

\section{The General Forms of the Equation of Motion}

The application of the principle of least action, taking into account the energy gauge by means of the cosmological constant within the framework of the covariant theory of gravitation and the four vector fields, leads to the equation for finding the metric tensor components [14]: 


$$
R^{\alpha \beta}-\frac{1}{4} R g^{\alpha \beta}=-\frac{1}{2 c k}\left(U^{\alpha \beta}+W^{\alpha \beta}+B^{\alpha \beta}+P^{\alpha \beta}\right)
$$

where $R^{\alpha \beta}$ is the Ricci tensor; $R$ is the scalar curvature; $g^{\alpha \beta}$ is the metric tensor; $c$ is the speed of light, $k=-\frac{c^{3}}{16 \pi G \beta}$, where $G$ is the gravitational constant; $\beta$ is a certain coefficient of the order of unity to be determined; $U^{\alpha \beta}, W^{\alpha \beta}, B^{\alpha \beta}$ and $P^{\alpha \beta}$ are the stress-energy tensors of the gravitational and electromagnetic fields, the acceleration field and the pressure field, respectively.

With the help of the covariant derivative $\nabla_{\beta}$ we can find the four-divergence of both parts of (1). The divergence of the left-hand side is equal to zero due to the equality to zero of the Einstein tensor's divergence, $\nabla_{\beta}\left(R^{\alpha \beta}-\frac{1}{2} R g^{\alpha \beta}\right)=0$, and also as a consequence of the fact that outside the body the scalar curvature vanishes, $R=0$, and inside the body it is considered to be a constant. The latter follows from the gauge condition of the system's energy [14]. The divergence of the right-hand side of (1) is also equal to zero:

$$
\nabla_{\beta}\left(U^{\alpha \beta}+W^{\alpha \beta}+B^{\alpha \beta}+P^{\alpha \beta}\right)=0
$$

Expression (2) for the space components of the tensors with the index $\alpha=1,2,3$ shows that the change over time of the fields' energy fluxes leads to the so-called fields' tensions. As for the time components of the tensors with the index $\alpha=0$, for them (2) is the expression of the generalized Poynting theorem for all the fields, both inside and outside the matter, that determines the energy balance in the system.

The stress-energy tensors of the gravitational field [10], [15], the electromagnetic field, the acceleration field and the pressure field [11], [14], presented in (2), are derived from the principle of least action:

$$
\begin{gathered}
U^{\alpha \beta}=-\frac{c^{2}}{4 \pi G} g^{\mu \kappa}\left(-g^{\alpha \lambda} g^{\sigma \beta}+\frac{1}{4} g^{\alpha \beta} g^{\sigma \lambda}\right) \Phi_{\mu \lambda} \Phi_{\kappa \sigma}, \\
W^{\alpha \beta}=\varepsilon_{0} c^{2} g^{\mu \kappa}\left(-g^{\alpha \lambda} g^{\sigma \beta}+\frac{1}{4} g^{\alpha \beta} g^{\sigma \lambda}\right) F_{\mu \lambda} F_{\kappa \sigma} . \\
B^{\alpha \beta}=\frac{c^{2}}{4 \pi \eta} g^{\mu \kappa}\left(-g^{\alpha \lambda} g^{\sigma \beta}+\frac{1}{4} g^{\alpha \beta} g^{\sigma \lambda}\right) u_{\mu \lambda} u_{\kappa \sigma}, \\
P^{\alpha \beta}=\frac{c^{2}}{4 \pi \sigma} g^{\mu \kappa}\left(-g^{\alpha \lambda} g^{\sigma \beta}+\frac{1}{4} g^{\alpha \beta} g^{\sigma \lambda}\right) f_{\mu \lambda} f_{\kappa \sigma} .
\end{gathered}
$$

Here $\Phi_{\mu \lambda}, F_{\mu \lambda}, u_{\mu \lambda}$ and $f_{\mu \lambda}$ represent the gravitational tensor, electromagnetic tensor, acceleration tensor and pressure field tensor, respectively; $\varepsilon_{0}$ is the electric constant, $\eta$ is the acceleration field coefficient; $\sigma$ is the pressure field coefficient.

All the tensors in (2) and in (3) are expressed in terms of the corresponding fields' strengths and solenoidal vectors. For example, the electromagnetic tensor $F_{\mu \lambda}$ and the stress-energy tensor of the electromagnetic field $W^{\alpha \beta}$ are expressed in terms of the field strength $\mathbf{E}$ and the magnetic field $\mathbf{B}$. 
Equation (2) inside the matter can be transformed in such a way that the field tensors themselves would appear in it instead of the stress-energy tensors. In particular, we can prove the following equalities $[15,16]$ :

$$
\begin{array}{cc}
\nabla_{\beta} U^{\alpha \beta}=-g^{\alpha \mu} \Phi_{\mu \nu} J^{v}, & \nabla_{\beta} W^{\alpha \beta}=-g^{\alpha \mu} F_{\mu \nu} j^{v}, \\
\nabla_{\beta} B^{\alpha \beta}=-g^{\alpha \mu} u_{\mu v} J^{v}, & \nabla_{\beta} P^{\alpha \beta}=-g^{\alpha \mu} f_{\mu \nu} J^{v} .
\end{array}
$$

In order to prove the validity of (4), it is necessary to substitute (3) into (4), to apply the covariant derivatives $\nabla_{\beta}$ to the products of the field tensors, and then to use the equations of the respective field, so that the mass four-current $J^{v}$ and the charge four-current $j^{v}$ would appear in (4).

In view of (4), equation (2) inside the matter turns into the equation of motion and takes the following form:

$$
\Phi_{\mu \nu} J^{v}+F_{\mu \nu} j^{v}+u_{\mu \nu} J^{v}+f_{\mu \nu} J^{v}=0
$$

The equation of motion in the form of (5) can also be obtained directly from the principle of least action [15], regardless of (1) and (2).

In order to reduce (5) to a more convenient form, we should take into account the expression for the mass current $J^{v}=\rho_{0} u^{v}$ in terms of the invariant mass density $\rho_{0}$ and the four-velocity $u^{v}$ of the particles, as well as the definition of the acceleration tensor $u_{\mu \nu}=\nabla_{\mu} U_{v}-\nabla_{v} U_{\mu}$, where $U_{v}$ is the four-potential of the acceleration field. With this in mind, we have the following:

$$
u_{\mu v} J^{v}=\rho_{0} u^{v}\left(\nabla_{\mu} U_{v}-\nabla_{v} U_{\mu}\right)=\rho_{0} u^{v} \nabla_{\mu} U_{v}-\rho_{0} \frac{D U_{\mu}}{D \tau} .
$$

In this case, the operator of proper-time-derivative $u^{v} \nabla_{v}=\frac{D}{D \tau}$ was used, where $D$ is the symbol of the four-differential in the curved spacetime, $\tau$ is the proper time [10]. The substitution of (6) into (5) gives the following:

$$
\rho_{0} \frac{D U_{\mu}}{D \tau}=\rho_{0} u^{v} \nabla_{\mu} U_{v}+\Phi_{\mu \nu} J^{v}+F_{\mu \nu} j^{v}+f_{\mu \nu} J^{v}
$$

If other vector fields are taken into account in the system, such as the dissipation field [12] or the macroscopic vector fields of strong and weak interactions [13], then the respective additional terms with the tensors of these fields appear on the right-hand side of (7).

For an arbitrary vector $B_{\mu}$ we can write:

$$
\nabla_{v} B_{\mu}=\partial_{\nu} B_{\mu}-\Gamma_{\mu \nu}^{\beta} B_{\beta}, \quad \frac{D B_{\mu}}{D \tau}=u^{v} \nabla_{v} B_{\mu}=u^{v} \partial_{v} B_{\mu}-u^{v} \Gamma_{\mu \nu}^{\beta} B_{\beta}=\frac{d B_{\mu}}{d \tau}-u^{v} \Gamma_{\mu \nu}^{\beta} B_{\beta},
$$

where $\Gamma_{\mu \nu}^{\beta}$ is the Christoffel symbol.

Replacement of $B_{\mu}$ by the four-potential of the acceleration field $U_{\mu}$ in (8) gives the following: 


$$
\frac{D U_{\mu}}{D \tau}=\frac{d U_{\mu}}{d \tau}-u^{v} \Gamma_{\mu \nu}^{\beta} U_{\beta}, \quad \nabla_{\mu} U_{v}=\partial_{\mu} U_{v}-\Gamma_{\mu \nu}^{\beta} U_{\beta}
$$

Let us substitute both equalities (9) into (7):

$$
\rho_{0} \frac{d U_{\mu}}{d \tau}=\rho_{0} u^{v} \partial_{\mu} U_{v}+\Phi_{\mu \nu} J^{v}+F_{\mu \nu} j^{v}+f_{\mu \nu} J^{v}
$$

We will divide equation (10) into two equations, one for the time components and the other for the space vector components, with the index $i=1,2,3$ :

$$
\begin{aligned}
& \rho_{0} \frac{d U_{0}}{d \tau}=\frac{\rho_{0}}{c} u^{v} \frac{\partial U_{v}}{\partial t}+\Phi_{0 v} J^{v}+F_{0 v} j^{v}+f_{0 v} J^{v} \\
& \rho_{0} \frac{d U_{i}}{d \tau}=\rho_{0} u^{v} \partial_{i} U_{v}+\Phi_{i v} J^{v}+F_{i v} j^{v}+f_{i v} J^{v}
\end{aligned}
$$

In (11), differentiation with respect to the proper time $\tau$ can be replaced by differentiation with respect to the coordinate time $t$ as follows: $\frac{d}{d \tau}=\frac{d t}{d \tau} \frac{d}{d t}$. In this case, for the mass four-current $J^{v}$ and for the charge four-current $j^{v}$, we can write:

$$
J^{v}=\rho_{0} u^{v}=\rho_{0} \frac{d x^{v}}{d \tau}=\rho_{0} \frac{d t}{d \tau} \frac{d x^{v}}{d t}=\rho_{0} \frac{d t}{d \tau}(c, \mathbf{v}), \quad j^{v}=\rho_{0 q} u^{v}=\rho_{0 q} \frac{d t}{d \tau}(c, \mathbf{v})
$$

where $\rho_{0 q}$ is the invariant charge density, $\mathbf{v}$ is the velocity of the matter particles' motion. In the Cartesian space coordinates, the four-dimensional quantity $(c, \mathbf{v})=\left(c, v^{1}, v^{2}, v^{3}\right)=\left(c, v_{x}, v_{y}, v_{z}\right)$ is expressed in terms of the speed of light and the components of the particles' velocity.

Also taking into account that the field tensors in (11) are expressed in terms of the strengths and solenoidal vectors, and the four-potential of the acceleration field $U_{\mu}=\left(\frac{\vartheta}{c},-\mathbf{U}\right)$ is defined by the scalar potential $\vartheta$ and the vector potential $\mathbf{U}$, in view of (12), we find the following:

$$
\begin{gathered}
\frac{d \vartheta}{d t}=\frac{d x^{v}}{d t} \frac{\partial U_{v}}{\partial t}+\boldsymbol{\Gamma} \cdot \mathbf{v}+\frac{\rho_{0 q}}{\rho_{0}} \mathbf{E} \cdot \mathbf{v}+\mathbf{C} \cdot \mathbf{v} . \\
\frac{d \mathbf{U}}{d t}=-\frac{d x^{v}}{d t} \partial_{i} U_{v}+\boldsymbol{\Gamma}+[\mathbf{v} \times \mathbf{\Omega}]+\frac{\rho_{0 q}}{\rho_{0}}(\mathbf{E}+[\mathbf{v} \times \mathbf{B}])+\mathbf{C}+[\mathbf{v} \times \mathbf{I}],
\end{gathered}
$$

where $\boldsymbol{\Gamma}, \mathbf{E}$ and $\mathbf{C}$ denote the strengths of the gravitational field, electromagnetic field and pressure field, respectively; and, similarly, $\boldsymbol{\Omega}$ is the gravitational torsion field; $\mathbf{B}$ is the magnetic induction, and $\mathbf{I}$ is the solenoidal vector of the pressure field.

According to the definition of the acceleration field tensor, its components include the strength $\mathbf{S}$ and the solenoidal vector $\mathbf{N}$, expressed in terms of the acceleration field potentials: 


$$
\mathbf{S}=-\nabla \vartheta-\frac{\partial \mathbf{U}}{\partial t}, \quad \mathbf{N}=\nabla \times \mathbf{U}
$$

The vectors $\mathbf{S}$ and $\mathbf{N}$ can be included in equations (13). To do this, on the left-hand side of (13) we must take into account the operator equation $\frac{d}{d t}=\frac{\partial}{\partial t}+\mathbf{v} \cdot \nabla$, and on the right-hand side of (13) we must expand the products of the four-vectors into their components, taking into account the definitions $x^{v}=(c t, \mathbf{r})$ and $\frac{d x^{v}}{d t}=(c, \mathbf{v})$ :

$$
\begin{gathered}
\frac{d x^{v}}{d t} \frac{\partial U_{v}}{\partial t}=\frac{\partial \vartheta}{\partial t}-v_{x} \frac{\partial U_{x}}{\partial t}-v_{y} \frac{\partial U_{y}}{\partial t}-v_{z} \frac{\partial U_{z}}{\partial t}=\frac{\partial \vartheta}{\partial t}-\mathbf{v} \cdot \frac{\partial \mathbf{U}}{\partial t} \\
(\mathbf{v} \cdot \nabla) \mathbf{U}+\frac{d x^{v}}{d t} \partial_{i} U_{v}=\nabla \vartheta-\mathbf{v} \times[\nabla \times \mathbf{U}]=\nabla \vartheta-\mathbf{v} \times \mathbf{N} .
\end{gathered}
$$

With this in mind, it follows from (13):

$$
\begin{gathered}
\mathbf{S} \cdot \mathbf{v}+\boldsymbol{\Gamma} \cdot \mathbf{v}+\frac{\rho_{0 q}}{\rho_{0}} \mathbf{E} \cdot \mathbf{v}+\mathbf{C} \cdot \mathbf{v}=0 . \\
\mathbf{S}+[\mathbf{v} \times \mathbf{N}]+\boldsymbol{\Gamma}+[\mathbf{v} \times \mathbf{\Omega}]+\frac{\rho_{0 q}}{\rho_{0}}(\mathbf{E}+[\mathbf{v} \times \mathbf{B}])+\mathbf{C}+[\mathbf{v} \times \mathbf{I}]=0 .
\end{gathered}
$$

If in (14) we multiply scalarly the second equation by the velocity $\mathbf{v}$, we will obtain the first equation in (14).

Equations (10), (11), (13) and (14), written in a covariant way, do not contain Christoffel symbols, which simplifies the solution of these equations. We should note that equations (14) are completely symmetric with respect to all the four vector fields. This means that, for example, if the field strengths and the velocity $\mathbf{v}$ of the system's typical particles are known as functions of time and coordinates, then from the first equation (14) we can find the relationship between the field coefficients, that is, between $\eta, G, \varepsilon_{0}$ and $\sigma$.

\section{Rectilinear Motion of an Ideal Solid Body}

An ideal solid body can be considered as the limiting case of the relativistic uniform system, in which the system's particles are fixed relative to each other. This means that the Lorentz factor $\gamma^{\prime}=\frac{1}{\sqrt{1-v^{\prime 2} / c^{2}}}$ of the particles is equal to 1 , and the velocity $\mathbf{v}^{\prime}$ of their motion is equal to zero in the body's center-of-momentum frame. This fact allows us to substantially simplify the equation of motion of the body.

We will start with the definition of the four-potential of the acceleration field of an arbitrary physical system. According to [11], the four-potential of any vector field, the vector potential of which is equal to zero in its proper reference frame, that is, in the center-of-momentum frame, in case of rectilinear motion in the laboratory reference frame can be represented by the formula:

$$
L_{\alpha}=\frac{k_{f} \varepsilon}{\rho_{0} c^{2}} u_{\alpha L}
$$


where $k_{f}=\frac{\rho_{0}}{\rho_{0 q}}$ for the electromagnetic field and $k_{f}=1$ for the other fields; $\varepsilon$ is the invariant energy density of the interaction between the field's four-potential and the corresponding four-current; $u_{\alpha L}$ is the four-velocity with a covariant index that specifies the motion of the center of momentum of the physical system in the laboratory reference frame.

In the proper reference frame $u_{\alpha L}=\left(c \frac{d t}{d \tau}, 0,0,0\right)$, and the vector potential as the space component of $L_{\alpha}$ vanishes according to (15). However, some physical systems, even if they have their own center of momentum fixed, have not only a scalar field potential but also a vector field potential within the system. Therefore, a more general expression for the field's four-potential in the laboratory reference frame is as follows:

$$
L_{\alpha}=M_{\alpha}^{\beta} L_{\beta}^{\prime},
$$

where $M_{\alpha}{ }^{\beta}$ is a matrix connecting the coordinates and time of the two reference frames, one of which is laboratory reference frame and the other moves together with the center of momentum of the physical system under consideration, so that there is the field's four-potential $L_{\beta}^{\prime}$ in it. In the special case of the system's motion at the constant velocity the matrix $M_{\alpha}{ }^{\beta}$ represents the Lorentz transformation matrix [10].

Let us now assume the simplest case, when the physical system in the form of a sphere has no general rotation, the typical particles of the system move randomly and they also have neither proper rotation nor their own vector potentials in the particles' center-of-momentum frames. In this case, we can use a simpler formula (15) instead of (16) to determine the four-potential of the acceleration field in case of rectilinear motion.

In a fixed sphere, the energy density in the volume of each particle $\varepsilon=\gamma^{\prime} \rho_{0} c^{2}$, and, according to (15), for the acceleration field, when the sphere moves in the laboratory reference frame, the fourpotential will equal $U_{\alpha L}=\gamma^{\prime} u_{\alpha L}$. This means that if for an observer inside the sphere with particles, within the framework of the relativistic uniform model, the quantity $\gamma^{\prime}$ is an invariantly defined Lorentz factor as a certain function of coordinates and time, then for an observer in the laboratory reference frame, in which the center of the sphere has the four-velocity $u_{\alpha L}$, the four-potential of the acceleration field for each point inside the moving sphere will equal $U_{\alpha L}$.

By definition, the four-potential of the acceleration field is the four-vector $U_{\mu}=\left(\frac{\vartheta}{c},-\mathbf{U}\right)$, where $\vartheta$ and $\mathbf{U}$ denote the scalar and vector potentials, respectively. Taking into account (15) and the relation $\varepsilon=\gamma^{\prime} \rho_{0} c^{2}$, it turns out that in the relativistic uniform system in the form of a fixed sphere the scalar potential will be $\vartheta=\gamma^{\prime} c^{2}$. As for the global vector potential of the acceleration field $\mathbf{U}$, it will differ from zero only in the systems with directed fluxes of particles.

In the ideal case, when the system of particles is not a rotating perfectly solid body and the particles inside the system are motionless, it must be $\gamma^{\prime}=1$, and then the four-potential of the acceleration field would coincide with the four-velocity of the system's center of momentum, $U_{\alpha L}=u_{\alpha L}$. A material point represents a miniature physical system, and if we do not go deeply into the structure of the internal motion of its matter and if we consider this point as a solid body, then the four-potential of the acceleration field of such a point will also be equal to the four-velocity of its motion.

Thus, in case of rectilinear motion of an ideal solid body in the absence of rotation, in equation of motion (7) the four-potential of the acceleration field $U_{\mu}$ can be replaced by the four-velocity of the 
body's center of momentum $u_{\mu}$. Taking into account the relation $u^{v} \nabla_{\mu} u_{v}=0$, which follows from the equality $\nabla_{\mu} c^{2}=\nabla_{\mu}\left(u_{v} u^{v}\right)=2 u^{\nu} \nabla_{\mu} u_{v}=0$, instead of (7) we obtain the following:

$$
\rho_{0} \frac{D u_{\mu}}{D \tau}=\rho_{0} a_{\mu}=\Phi_{\mu \nu} J^{v}+F_{\mu \nu} j^{v}+f_{\mu \nu} J^{v}
$$

On the left-hand side of (17) there is the covariant four-acceleration $a_{\mu}=\frac{D u_{\mu}}{D \tau}$, and on the right-hand side there is the sum of the densities of the gravitational and electromagnetic forces and the density of the pressure force. In particular, the expression $F_{\mu \nu} j^{v}$ defines the density of the electromagnetic Lorentz force. Equations (10) and (11), in view the relation $u^{v} \partial_{\mu} u_{v}=0$, are written as follows:

$$
\begin{gathered}
\rho_{0} \frac{d u_{\mu}}{d \tau}=\Phi_{\mu \nu} J^{v}+F_{\mu \nu} j^{v}+f_{\mu \nu} J^{v} . \\
\rho_{0} \frac{d u_{0}}{d \tau}=\Phi_{0 v} J^{v}+F_{0 v} j^{v}+f_{0 v} J^{v}, \quad \rho_{0} \frac{d u_{i}}{d \tau}=\Phi_{i v} J^{v}+F_{i v} j^{v}+f_{i v} J^{v} .
\end{gathered}
$$

On the left-hand side of (18) we will use the expression for the four-velocity $u_{\mu}=\frac{d x_{\mu}}{d \tau}=\frac{d t}{d \tau} \frac{d x_{\mu}}{d t}=\frac{d t}{d \tau}(c,-\mathbf{v})$, and on the right-hand side we will express the field tensors in terms of the strengths and solenoidal vectors. Then, in view of (12), equation (18) can be transformed as follows:

$$
\begin{gathered}
c^{2} \frac{d}{d t}\left(\frac{d t}{d \tau}\right)=\boldsymbol{\Gamma} \cdot \mathbf{v}+\frac{\rho_{0 q}}{\rho_{0}} \mathbf{E} \cdot \mathbf{v}+\mathbf{C} \cdot \mathbf{v}, \\
\frac{d}{d t}\left(\frac{d t}{d \tau} \mathbf{v}\right)=\boldsymbol{\Gamma}+[\mathbf{v} \times \mathbf{\Omega}]+\frac{\rho_{0 q}}{\rho_{0}}(\mathbf{E}+[\mathbf{v} \times \mathbf{B}])+\mathbf{C}+[\mathbf{v} \times \mathbf{I}] .
\end{gathered}
$$

In the flat Minkowski spacetime $\frac{d t}{d \tau}=\gamma$, where $\gamma=\frac{1}{\sqrt{1-v^{2} / c^{2}}}$ is the Lorentz factor, the Christoffel symbols vanish, and the metric tensor $g_{\mu v}$ transforms into the metric tensor $\eta_{\mu \nu}$, which is independent of time and coordinates and has only the diagonal components. As a result, equations (19) are also simplified:

$$
\begin{gathered}
c^{2} \frac{d \gamma}{d t}=\boldsymbol{\Gamma} \cdot \mathbf{v}+\frac{\rho_{0 q}}{\rho_{0}} \mathbf{E} \cdot \mathbf{v}+\mathbf{C} \cdot \mathbf{v}, \\
\frac{d(\gamma \mathbf{v})}{d t}=\boldsymbol{\Gamma}+[\mathbf{v} \times \mathbf{\Omega}]+\frac{\rho_{0 q}}{\rho_{0}}(\mathbf{E}+[\mathbf{v} \times \mathbf{B}])+\mathbf{C}+[\mathbf{v} \times \mathbf{I}] .
\end{gathered}
$$

The three-dimensional acceleration vector of a solid body or a particle is $\mathbf{a}=\frac{d \mathbf{v}}{d t}$, so that $\frac{d(\gamma \mathbf{v})}{d t}=\gamma \mathbf{a}+\mathbf{v} \frac{d \gamma}{d t}$. Substituting here the expression $\frac{d \gamma}{d t}$ from (20) and comparing with (21), we 
obtain the equation for the acceleration in the framework of the special theory of relativity, expressed in terms of the fields' strengths and solenoidal vectors:

$$
\gamma \mathbf{a}=\boldsymbol{\Gamma}+[\mathbf{v} \times \mathbf{\Omega}]+\frac{\rho_{0 q}}{\rho_{0}}(\mathbf{E}+[\mathbf{v} \times \mathbf{B}])+\mathbf{C}+[\mathbf{v} \times \mathbf{I}]-\frac{1}{c^{2}} \mathbf{v}\left(\boldsymbol{\Gamma} \cdot \mathbf{v}+\frac{\rho_{0 q}}{\rho_{0}} \mathbf{E} \cdot \mathbf{v}+\mathbf{C} \cdot \mathbf{v}\right) .
$$

If we denote the force density as

$$
\mathbf{f}=\rho_{0}(\boldsymbol{\Gamma}+[\mathbf{v} \times \mathbf{\Omega}])+\rho_{0 q}(\mathbf{E}+[\mathbf{v} \times \mathbf{B}])+\rho_{0}(\mathbf{C}+[\mathbf{v} \times \mathbf{I}]),
$$

then $(20-22)$ can be rewritten as follows:

$$
\rho_{0} c^{2} \frac{d \gamma}{d t}=\mathbf{f} \cdot \mathbf{v}, \quad \rho_{0} \frac{d(\gamma \mathbf{v})}{d t}=\mathbf{f}, \quad \gamma \rho_{0} \mathbf{a}=\mathbf{f}-\frac{\mathbf{f} \cdot \mathbf{v}}{c^{2}} \mathbf{v}
$$

The relativistic energy and momentum of the solid body with the mass $m$ and charge $q$ are given by the expressions $W=\gamma m c^{2}, \mathbf{p}=\gamma m \mathbf{v}$. Let us assume that the mass and charge of the body are constant in motion. Then after multiplying equations (20) and (21) by the mass $m$, we can introduce the force $\mathbf{F}$ and represent the equations as follows:

$$
\begin{gathered}
\frac{d W}{d t}=m \boldsymbol{\Gamma} \cdot \mathbf{v}+q \mathbf{E} \cdot \mathbf{v}+m \mathbf{C} \cdot \mathbf{v}=\mathbf{F} \cdot \mathbf{v} \\
\frac{d \mathbf{p}}{d t}=m \boldsymbol{\Gamma}+m[\mathbf{v} \times \mathbf{\Omega}]+q \mathbf{E}+q[\mathbf{v} \times \mathbf{B}]+m \mathbf{C}+m[\mathbf{v} \times \mathbf{I}]=\mathbf{F}
\end{gathered}
$$

In the general case, the fields inside a solid body are non-uniform and differ in value at different points. In this case, the body can be divided into $N$ parts with the masses $m_{n}$, where the index $n$ ranges from 0 to $N$. Multiplying equations (20) and (21) by $m_{1}, m_{2} \ldots, m_{n}$, then summing the equations, and passing from the sums to the integrals over the whole body, we arrive at the following:

$$
\begin{aligned}
& \frac{d}{d t} \sum_{n=1}^{N}\left(\gamma m_{n} c^{2}\right)=\sum_{n=1}^{N}\left(m_{n} \boldsymbol{\Gamma} \cdot \mathbf{v}+q_{n} \mathbf{E} \cdot \mathbf{v}+m_{n} \mathbf{C} \cdot \mathbf{v}\right)=\int(\boldsymbol{\Gamma} \cdot \mathbf{v}+\mathbf{C} \cdot \mathbf{v}) d m+\int \mathbf{E} \cdot \mathbf{v} d q \\
& \quad \frac{d}{d t} \sum_{n=1}^{N}\left(\gamma m_{n} \mathbf{v}\right)=\sum_{n=1}^{N}\left(m_{n} \boldsymbol{\Gamma}+m_{n}[\mathbf{v} \times \mathbf{\Omega}]+q_{n} \mathbf{E}+q_{n}[\mathbf{v} \times \mathbf{B}]+m_{n} \mathbf{C}+m_{n}[\mathbf{v} \times \mathbf{I}]\right)= \\
& \quad=\int(\boldsymbol{\Gamma}+[\mathbf{v} \times \mathbf{\Omega}]+\mathbf{C}+[\mathbf{v} \times \mathbf{I}]) d m+\int(\mathbf{E}+[\mathbf{v} \times \mathbf{B}]) d q .
\end{aligned}
$$

Comparison with the previous equations gives the following:

$$
\begin{gathered}
W=\gamma m c^{2}=\sum_{n=1}^{N}\left(\gamma m_{n} c^{2}\right), \quad \mathbf{p}=\gamma m \mathbf{v}=\sum_{n=1}^{N}\left(\gamma m_{n} \mathbf{v}\right), \quad m=\sum_{n=1}^{N} m_{n}, \\
\mathbf{F}=\int(\boldsymbol{\Gamma}+[\mathbf{v} \times \mathbf{\Omega}]+\mathbf{C}+[\mathbf{v} \times \mathbf{I}]) d m+\int(\mathbf{E}+[\mathbf{v} \times \mathbf{B}]) d q .
\end{gathered}
$$


In these relations it is taken into account that in case of rectilinear motion of a solid body without rotation of this body, the velocity $\mathbf{v}$ and the Lorentz factor $\gamma$ are constant for all the points of the body.

The case is possible when the body does not rotate in the center-of-momentum frame, but it does not move along a straight line. This leads to the fact that the body rotates relative to the point located at the instantaneous radius of curvature of the motion trajectory, and then the velocities of the points inside the body become different. In this case, the motion of the body's points should be described by more general equations (14).

\section{Expression of the Equation of Motion in Terms of the Fields' Four-Potentials}

By definition, the field tensors are expressed in terms of the four-curl applied to the fields' fourpotentials. For the acceleration field, gravitational and electromagnetic fields and pressure field, this gives the following:

$$
\begin{array}{cc}
u_{\mu \nu}=\nabla_{\mu} U_{v}-\nabla_{\nu} U_{\mu}, & \Phi_{\mu v}=\nabla_{\mu} D_{v}-\nabla_{\nu} D_{\mu}, \\
F_{\mu v}=\nabla_{\mu} A_{v}-\nabla_{v} A_{\mu}, & f_{\mu v}=\nabla_{\mu} \pi_{v}-\nabla_{v} \pi_{\mu},
\end{array}
$$

where $U_{\mu}=\left(\frac{\vartheta}{c},-\mathbf{U}\right)$ is the four-potential of the acceleration field, $\vartheta$ and $\mathbf{U}$ denote the scalar and vector potentials, respectively,

$D_{\mu}=\left(\frac{\psi}{c},-\mathbf{D}\right)$ is the four-potential of the gravitational field, described in terms of the scalar potential $\psi$ and the vector potential $\mathbf{D}$ of this field,

$A_{\mu}=\left(\frac{\varphi}{c},-\mathbf{A}\right)$ is the four-potential of the electromagnetic field, given by the scalar potential $\varphi$ and the vector potential $\mathbf{A}$ of this field,

$\pi_{\mu}=\left(\frac{\wp}{c},-\boldsymbol{\Pi}\right)$ is the four-potential of the pressure field, containing the scalar potential $\wp$ and the vector potential $\Pi$.

Let us substitute (23) into (7), taking into account the expressions for the mass current $J^{v}=\rho_{0} u^{v}$ and for the charge current $j^{v}=\rho_{0 q} u^{v}$, and also taking into account rule (8) for the operator of the proper-time-derivative. We will also use the expression for the covariant derivative for the arbitrary four-vector $B_{v}$ :

$$
\nabla_{\mu} B_{v}=\partial_{\mu} B_{v}-\Gamma_{\mu \nu}^{\beta} B_{\beta}
$$

All this gives the following equation:

$$
\frac{d\left(U_{\mu}+D_{\mu}+\pi_{\mu}\right)}{d \tau}+\frac{\rho_{0 q}}{\rho_{0}} \frac{d A_{\mu}}{d \tau}=u^{v} \partial_{\mu} U_{v}+u^{v} \partial_{\mu} D_{v}+\frac{\rho_{0 q}}{\rho_{0}} u^{v} \partial_{\mu} A_{v}+u^{v} \partial_{\mu} \pi_{v}
$$

Equation of motion (24) falls into two equations, since it can be written separately for the time and space vector components of the fields' four-potentials. Using the expression $\partial_{0}=\frac{1}{c} \frac{\partial}{\partial t}$ for the time component of the partial derivative, we have: 


$$
\begin{aligned}
& \frac{d(\vartheta+\psi+\wp)}{d \tau}+\frac{\rho_{0 q}}{\rho_{0}} \frac{d \varphi}{d \tau}=u^{v} \frac{\partial U_{v}}{\partial t}+u^{v} \frac{\partial D_{v}}{\partial t}+\frac{\rho_{0 q}}{\rho_{0}} u^{v} \frac{\partial A_{v}}{\partial t}+u^{v} \frac{\partial \pi_{v}}{\partial t} \\
& \frac{d(\mathbf{U}+\mathbf{D}+\boldsymbol{\Pi})}{d \tau}+\frac{\rho_{0 q}}{\rho_{0}} \frac{d \mathbf{A}}{d \tau}=-u^{v} \partial_{i} U_{v}-u^{v} \partial_{i} D_{v}-\frac{\rho_{0 q}}{\rho_{0}} u^{v} \partial_{i} A_{v}-u^{v} \partial_{i} \pi_{v}
\end{aligned}
$$

In (25) the three-dimensional spatial gradient operator in Cartesian coordinates has the components $\partial_{i}=\left(\frac{\partial}{\partial x}, \frac{\partial}{\partial y}, \frac{\partial}{\partial z}\right)$, while the index $i=1,2,3$ in $\partial_{i}$ defines the components, which correspond to the components $\mathbf{U}=U_{i}, \mathbf{D}=D_{i}, \boldsymbol{\Pi}=\Pi_{i}$ and $\mathbf{A}=A_{i}$ of the vector field potentials.

The peculiarity of the covariant equations of motion (24) and (25) is the fact that they are fully expressed in terms of the fields' four-potentials or in terms of their components in the form of the fields' scalar and vector potentials. It is to be recalled that, in contrast, the equations of motion in the form of (7), (10) and (11) are expressed in terms of tensors, that is, in terms of the fields' strengths and solenoidal vectors, as was shown in (13) and (14).

We will use the relation $\frac{d}{d \tau}=\frac{d t}{d \tau} \frac{d}{d t}$ in equations (25). This gives the following:

$$
\begin{gathered}
\frac{d(\vartheta+\psi+\wp)}{d t}+\frac{\rho_{0 q}}{\rho_{0}} \frac{d \varphi}{d t}=\frac{d x^{v}}{d t} \frac{\partial U_{v}}{\partial t}+\frac{d x^{v}}{d t} \frac{\partial D_{v}}{\partial t}+\frac{\rho_{0 q}}{\rho_{0}} \frac{d x^{v}}{d t} \frac{\partial A_{v}}{\partial t}+\frac{d x^{v}}{d t} \frac{\partial \pi_{v}}{\partial t} \\
\frac{d(\mathbf{U}+\mathbf{D}+\mathbf{\Pi})}{d t}+\frac{\rho_{0 q}}{\rho_{0}} \frac{d \mathbf{A}}{d t}=-\frac{d x^{v}}{d t} \partial_{i} U_{v}-\frac{d x^{v}}{d t} \partial_{i} D_{v}-\frac{\rho_{0 q}}{\rho_{0}} \frac{d x^{v}}{d t} \partial_{i} A_{v}-\frac{d x^{v}}{d t} \partial_{i} \pi_{v} .
\end{gathered}
$$

For the case of rectilinear motion of an ideal solid body without rotation, the four-potential $U_{\mu}$ of the acceleration field at each point of the body coincides with the four-velocity $u_{\mu}$ of motion of the body's center of momentum. In this case in (25), (26) and (27) we will have $\vartheta=c^{2} \frac{d t}{d \tau}, \mathbf{U}=\frac{d t}{d \tau} \mathbf{v}$.

In the Minkowski spacetime $\frac{d t}{d \tau}=\gamma$ and the equations of motion of a solid body are further simplified, since then in (26) and (27) $\vartheta=\gamma c^{2}, \mathbf{U}=\gamma \mathbf{v}, U_{v}=u_{v}=(\gamma c,-\gamma \mathbf{v})$. In addition, since $\gamma^{2}=\frac{1}{1-v^{2} / c^{2}}$, the following relations hold true:

$$
\begin{gathered}
\frac{d x^{v}}{d t} \frac{\partial u_{v}}{\partial t}=c^{2} \frac{\partial \gamma}{\partial t}-\frac{1}{2 \gamma} \frac{\partial\left(\gamma^{2} v^{2}\right)}{\partial t}=\frac{1}{2 \gamma}\left[\frac{\partial\left(\gamma^{2} c^{2}\right)}{\partial t}-\frac{\partial\left(\gamma^{2} v^{2}\right)}{\partial t}\right]=0, \\
-\frac{d x^{v}}{d t} \partial_{i} u_{v}=-c^{2} \partial_{i} \gamma+\frac{1}{2 \gamma} \partial_{i}\left(\gamma^{2} v^{2}\right)=-\frac{1}{2 \gamma}\left[\partial_{i}\left(\gamma^{2} c^{2}\right)-\partial_{i}\left(\gamma^{2} v^{2}\right)\right]=0 .
\end{gathered}
$$

Taking this into account, instead of (26) and (27) we can write the following:

$$
c^{2} \frac{d \gamma}{d t}=-\frac{d(\psi+\wp)}{d t}-\frac{\rho_{0 q}}{\rho_{0}} \frac{d \varphi}{d t}+\frac{d x^{v}}{d t} \frac{\partial D_{v}}{\partial t}+\frac{\rho_{0 q}}{\rho_{0}} \frac{d x^{v}}{d t} \frac{\partial A_{v}}{\partial t}+\frac{d x^{v}}{d t} \frac{\partial \pi_{v}}{\partial t},
$$




$$
\frac{d(\gamma \mathbf{v})}{d t}=-\frac{d(\mathbf{D}+\boldsymbol{\Pi})}{d t}-\frac{\rho_{0 q}}{\rho_{0}} \frac{d \mathbf{A}}{d t}-\frac{d x^{v}}{d t} \partial_{i} D_{v}-\frac{\rho_{0 q}}{\rho_{0}} \frac{d x^{v}}{d t} \partial_{i} A_{v}-\frac{d x^{v}}{d t} \partial_{i} \pi_{v} .
$$

\section{Complementarity of Description in Terms of the Potentials and Field Strengths}

We will demonstrate the equivalence of equations of motion (26), (27) and equations (14). For this we will need expressions (23) written in a covariant vector form. The field tensors' components according to (23) are the strengths and solenoidal vectors, which by definition depend on the time and space derivatives of the corresponding scalar and vector potentials:

$$
\begin{array}{r}
\boldsymbol{\Gamma}=-\nabla \psi-\frac{\partial \mathbf{D}}{\partial t}, \quad \mathbf{\Omega}=\nabla \times \mathbf{D}, \quad \mathbf{E}=-\nabla \varphi-\frac{\partial \mathbf{A}}{\partial t}, \quad \mathbf{B}=\nabla \times \mathbf{A}, \\
\mathbf{S}=-\nabla \vartheta-\frac{\partial \mathbf{U}}{\partial t}, \quad \mathbf{N}=\nabla \times \mathbf{U}, \quad \mathbf{C}=-\nabla \wp-\frac{\partial \boldsymbol{\Pi}}{\partial t}, \quad \mathbf{I}=\nabla \times \boldsymbol{\Pi} .
\end{array}
$$

We will use the operator equation $\frac{d}{d t}=\frac{\partial}{\partial t}+\mathbf{v} \cdot \nabla$ on the left-hand side of equation (26), and on the right-hand side of (26), in view of the definitions $x^{v}=(c t, \mathbf{r})$ and $\frac{d x^{v}}{d t}=(c, \mathbf{v})$, we will expand the products of the four-vectors into their components, so we will obtain, for example, the following:

$$
\frac{d x^{v}}{d t} \frac{\partial D_{v}}{\partial t}=\frac{\partial \psi}{\partial t}-v_{x} \frac{\partial D_{x}}{\partial t}-v_{y} \frac{\partial D_{y}}{\partial t}-v_{z} \frac{\partial D_{z}}{\partial t}=\frac{\partial \psi}{\partial t}-\mathbf{v} \cdot \frac{\partial \mathbf{D}}{\partial t}
$$

Transforming also the remaining terms on the right-hand side of (26), we arrive at the following:

$$
\mathbf{v} \cdot \nabla(\vartheta+\psi+\wp)+\frac{\rho_{0 q}}{\rho_{0}} \mathbf{v} \cdot \nabla \varphi=-\mathbf{v} \cdot \frac{\partial \mathbf{U}}{\partial t}-\mathbf{v} \cdot \frac{\partial \mathbf{D}}{\partial t}-\frac{\rho_{0 q}}{\rho_{0}} \mathbf{v} \cdot \frac{\partial \mathbf{A}}{\partial t}-\mathbf{v} \cdot \frac{\partial \mathbf{\Pi}}{\partial t}
$$

Now we can take into account (30):

$$
\mathbf{v} \cdot \mathbf{S}+\mathbf{v} \cdot \boldsymbol{\Gamma}+\frac{\rho_{0 q}}{\rho_{0}} \mathbf{v} \cdot \mathbf{E}+\mathbf{v} \cdot \mathbf{C}=0
$$

This equality coincides with the first equality in (14).

Taking now the Cartesian coordinates for simplifying, we will project all the terms in (27) onto the axis $O X$, so that in the equation we will have $i=1, \partial_{1}=\partial_{x}=\frac{\partial}{\partial x}$ :

$$
\frac{d\left(U_{x}+D_{x}+\Pi_{x}\right)}{d t}+\frac{\rho_{0 q}}{\rho_{0}} \frac{d A_{x}}{d t}=-\frac{d x^{v}}{d t} \frac{\partial U_{v}}{\partial x}-\frac{d x^{v}}{d t} \frac{\partial D_{v}}{\partial x}-\frac{\rho_{0 q}}{\rho_{0}} \frac{d x^{v}}{d t} \frac{\partial A_{v}}{\partial x}-\frac{d x^{v}}{d t} \frac{\partial \pi_{v}}{\partial x}
$$

In view of (30), we will expand the products of the four-vectors into their components on the righthand side of (31): 


$$
\begin{gathered}
-\frac{d x^{v}}{d t} \frac{\partial D_{v}}{\partial x}=-\frac{\partial \psi}{\partial x}+v_{x} \frac{\partial D_{x}}{\partial x}+v_{y} \frac{\partial D_{y}}{\partial x}+v_{z} \frac{\partial D_{z}}{\partial x}=\Gamma_{x}+\frac{\partial D_{x}}{\partial t}+v_{x} \frac{\partial D_{x}}{\partial x}+[\mathbf{v} \times \mathbf{\Omega}]_{x}+v_{y} \frac{\partial D_{x}}{\partial y}+v_{z} \frac{\partial D_{x}}{\partial z}= \\
=\Gamma_{x}+\frac{\partial D_{x}}{\partial t}+[\mathbf{v} \times \mathbf{\Omega}]_{x}+(\mathbf{v} \cdot \nabla) D_{x}=\Gamma_{x}+\frac{d D_{x}}{d t}+[\mathbf{v} \times \mathbf{\Omega}]_{x} . \\
-\frac{\rho_{0 q}}{\rho_{0}} \frac{d x^{v}}{d t} \frac{\partial A_{v}}{\partial x}=\frac{\rho_{0 q}}{\rho_{0}} E_{x}+\frac{\rho_{0 q}}{\rho_{0}} \frac{d A_{x}}{d t}+\frac{\rho_{0 q}}{\rho_{0}}[\mathbf{v} \times \mathbf{B}]_{x}, \quad-\frac{d x^{v}}{d t} \frac{\partial \pi_{v}}{\partial x}=C_{x}+\frac{d \Pi_{x}}{d t}+[\mathbf{v} \times \mathbf{I}]_{x}, \\
-\frac{d x^{v}}{d t} \frac{\partial U_{v}}{\partial x}=S_{x}+\frac{d U_{x}}{d t}+[\mathbf{v} \times \mathbf{N}]_{x} .
\end{gathered}
$$

Substitution of all this into (31) gives the following:

$$
S_{x}+[\mathbf{v} \times \mathbf{N}]_{x}+\Gamma_{x}+[\mathbf{v} \times \mathbf{\Omega}]_{x}+\frac{\rho_{0 q}}{\rho_{0}} E_{x}+\frac{\rho_{0 q}}{\rho_{0}}[\mathbf{v} \times \mathbf{B}]_{x}+C_{x}+[\mathbf{v} \times \mathbf{I}]_{x}=0
$$

This equation coincides with the projection of the second equation in (14) onto the axis $O X$. Thus, equations of motion (26) and (27) are equivalent to equations (14), which is a consequence of the relation between the strengths and the field potentials in (30).

In addition to the presence of proper equations of motion, the complementarity of the potentials and the field strengths is emphasized by the fact that for them there are proper field equations. Thus, for the four fields used here, the field equations for determining the strengths and the solenoidal vectors have the following form [14]:

$$
\begin{array}{cc}
\nabla_{\beta} \Phi^{\alpha \beta}=\frac{4 \pi G}{c^{2}} J^{\alpha}, & \nabla_{\sigma} \Phi_{\mu \nu}+\nabla_{v} \Phi_{\sigma \mu}+\nabla_{\mu} \Phi_{v \sigma}=0, \\
\nabla_{\beta} F^{\alpha \beta}=-\mu_{0} j^{\alpha}, & \nabla_{\sigma} F_{\mu \nu}+\nabla_{\nu} F_{\sigma \mu}+\nabla_{\mu} F_{v \sigma}=0, \\
\nabla_{\beta} u^{\alpha \beta}=-\frac{4 \pi \eta}{c^{2}} J^{\alpha}, & \nabla_{\sigma} u_{\mu \nu}+\nabla_{v} u_{\sigma \mu}+\nabla_{\mu} u_{v \sigma}=0, \\
\nabla_{\beta} f^{\alpha \beta}=-\frac{4 \pi \sigma}{c^{2}} J^{\alpha}, & \nabla_{\sigma} f_{\mu \nu}+\nabla_{v} f_{\sigma \mu}+\nabla_{\mu} f_{v \sigma}=0 .
\end{array}
$$

In the first equations in (32), the field tensors can be replaced by their expressions in terms of the four-potentials according to (23). After this, we should use the rule for the difference of the secondorder covariant derivatives of the arbitrary four-vector $B^{\beta}$ :

$$
\nabla_{\beta} \nabla^{\alpha} B^{\beta}=\nabla^{\alpha} \nabla_{\beta} B^{\beta}-B^{\mu} R_{\mu}^{\alpha} .
$$

where $R_{\mu}{ }^{\alpha}$ is the Ricci tensor with the mixed indices.

With this in mind, we arrive at the field equations for the four-potentials of the fields:

$$
\begin{array}{cc}
\nabla_{\beta} \nabla^{\beta} D^{\alpha}=-\frac{4 \pi G}{c^{2}} J^{\alpha}+\nabla^{\alpha} \nabla_{\beta} D^{\beta}-D^{\mu} R_{\mu}{ }^{\alpha}, & \nabla_{\beta} \nabla^{\beta} A^{\alpha}=\mu_{0} j^{\alpha}+\nabla^{\alpha} \nabla_{\beta} A^{\beta}-A^{\mu} R_{\mu}{ }^{\alpha} . \\
\nabla_{\beta} \nabla^{\beta} U^{\alpha}=\frac{4 \pi \eta}{c^{2}} J^{\alpha}+\nabla^{\alpha} \nabla_{\beta} U^{\beta}-U^{\mu} R_{\mu}{ }^{\alpha}, & \nabla_{\beta} \nabla^{\beta} \pi^{\alpha}=\frac{4 \pi \sigma}{c^{2}} J^{\alpha}+\nabla^{\alpha} \nabla_{\beta} \pi^{\beta}-\pi^{\mu} R_{\mu}{ }^{\alpha} .
\end{array}
$$


As a rule, the four-potentials of the fields are gauged in such a way that their covariant divergences are equal to zero:

$$
\nabla_{\beta} D^{\beta}=0, \quad \nabla_{\beta} A^{\beta}=0, \quad \nabla_{\beta} U^{\beta}=0, \quad \nabla_{\beta} \pi^{\beta}=0 .
$$

The use of (34) leads to simplification of (33):

$$
\begin{array}{cc}
\nabla_{\beta} \nabla^{\beta} D^{\alpha}=-\frac{4 \pi G}{c^{2}} J^{\alpha}-D^{\mu} R_{\mu}{ }^{\alpha}, & \nabla_{\beta} \nabla^{\beta} A^{\alpha}=\mu_{0} j^{\alpha}-A^{\mu} R_{\mu}{ }^{\alpha} . \\
\nabla_{\beta} \nabla^{\beta} U^{\alpha}=\frac{4 \pi \eta}{c^{2}} J^{\alpha}-U^{\mu} R_{\mu}{ }^{\alpha}, & \nabla_{\beta} \nabla^{\beta} \pi^{\alpha}=\frac{4 \pi \sigma}{c^{2}} J^{\alpha}-\pi^{\mu} R_{\mu}{ }^{\alpha} .
\end{array}
$$

It is interesting that the equations with the d'Alembertian $\nabla_{\beta} \nabla^{\beta}$ can be obtained not only for the four-potentials, but also for the field tensors themselves. The derivation of one such equation is shown in Appendix A using the example of the electromagnetic field equations in (25). According to the relation (A10), we obtain the following:

$$
\nabla^{\sigma} \nabla_{\sigma} F_{\mu v}=\mu_{0} \nabla_{\mu} j_{v}-\mu_{0} \nabla_{v} j_{\mu}+F_{v \rho} R_{\mu}^{\rho}-F_{\mu \rho} R_{v}^{\rho}+R_{\mu v, \lambda \eta} F^{\eta \lambda}
$$

Similarly, we can write for the tensors of the gravitational field, the acceleration field and the pressure field:

$$
\begin{gathered}
\nabla^{\sigma} \nabla_{\sigma} \Phi_{\mu v}=-\frac{4 \pi G}{c^{2}} \nabla_{\mu} J_{v}+\frac{4 \pi G}{c^{2}} \nabla_{v} J_{\mu}+\Phi_{v \rho} R_{\mu}^{\rho}-\Phi_{\mu \rho} R_{v}^{\rho}+R_{\mu v, \lambda \eta} \Phi^{\eta \lambda} \\
\nabla^{\sigma} \nabla_{\sigma} U_{\mu v}=\frac{4 \pi \eta}{c^{2}} \nabla_{\mu} J_{v}-\frac{4 \pi \eta}{c^{2}} \nabla_{v} J_{\mu}+U_{v \rho} R_{\mu}^{\rho}-U_{\mu \rho} R_{v}^{\rho}+R_{\mu v, \lambda \eta} U^{\eta \lambda}, \\
\nabla^{\sigma} \nabla_{\sigma} f_{\mu v}=\frac{4 \pi \sigma}{c^{2}} \nabla_{\mu} J_{v}-\frac{4 \pi \sigma}{c^{2}} \nabla_{v} J_{\mu}+f_{v \rho} R_{\mu}^{\rho}-f_{\mu \rho} R_{v}^{\rho}+R_{\mu v, \lambda \eta} f^{\eta \lambda}
\end{gathered}
$$

Equations (36) represent the wave equations for the field tensors in the curved spacetime.

In the Minkowski spacetime, the covariant derivative becomes a four-gradient, the covariant d'Alembertian $\quad \nabla_{\beta} \nabla^{\beta}=\nabla^{\sigma} \nabla_{\sigma}$ is transformed into the ordinary d'Alembertian $\partial_{\beta} \partial^{\beta}=\frac{1}{c^{2}} \frac{\partial^{2}}{\partial t^{2}}-\Delta=\frac{1}{c^{2}} \frac{\partial^{2}}{\partial t^{2}}-\frac{\partial^{2}}{\partial x^{2}}-\frac{\partial^{2}}{\partial y^{2}}-\frac{\partial^{2}}{\partial z^{2}}$, the Ricci tensor $R_{\mu}{ }^{\alpha}$ and the curvature tensor $R_{\mu v, \lambda \eta}$ vanish. In this case, equations (32) can be presented as the equations for determining the fields' strengths and the solenoidal vectors:

$$
\begin{gathered}
\nabla \cdot \boldsymbol{\Gamma}=-4 \pi G \gamma \rho_{0}, \quad \nabla \times \boldsymbol{\Omega}=\frac{1}{c^{2}} \frac{\partial \boldsymbol{\Gamma}}{\partial t}-\frac{4 \pi G \mathbf{J}}{c^{2}}, \quad \nabla \cdot \boldsymbol{\Omega}=0, \quad \nabla \times \boldsymbol{\Gamma}=-\frac{\partial \boldsymbol{\Omega}}{\partial t} . \\
\nabla \cdot \mathbf{E}=\mu_{0} \gamma \rho_{0 q}, \quad \nabla \times \mathbf{B}=\frac{1}{c^{2}} \frac{\partial \mathbf{E}}{\partial t}+\frac{\mu_{0} \mathbf{j}}{c^{2}}, \quad \nabla \cdot \mathbf{B}=0, \quad \nabla \times \mathbf{E}=-\frac{\partial \mathbf{B}}{\partial t} .
\end{gathered}
$$




$$
\begin{gathered}
\nabla \cdot \mathbf{S}=4 \pi \eta \gamma \rho_{0}, \quad \nabla \times \mathbf{N}=\frac{1}{c^{2}} \frac{\partial \mathbf{S}}{\partial t}+\frac{4 \pi \eta \mathbf{J}}{c^{2}}, \quad \nabla \cdot \mathbf{N}=0, \quad \nabla \times \mathbf{S}=-\frac{\partial \mathbf{N}}{\partial t} . \\
\nabla \cdot \mathbf{C}=4 \pi \sigma \gamma \rho_{0}, \quad \nabla \times \mathbf{I}=\frac{1}{c^{2}} \frac{\partial \mathbf{C}}{\partial t}+\frac{4 \pi \sigma \mathbf{J}}{c^{2}}, \quad \nabla \cdot \mathbf{I}=0, \quad \nabla \times \mathbf{C}=-\frac{\partial \mathbf{I}}{\partial t} .
\end{gathered}
$$

In (37) $\mathbf{j}=\gamma \rho_{0 q} \mathbf{v}$ is the electric current density, $\gamma$ is the Lorentz factor, $\rho_{0 q}$ is the invariant charge density, $\mathbf{v}$ is the velocity of motion of the matter's particles, and $\mathbf{J}=\gamma \rho_{0} \mathbf{v}$ is the mass current density.

In this case, equations (35) turn into the wave equations for the four-potentials in the framework of the special theory of relativity. We can expand these equations and write them separately for the scalar and vector potentials:

$$
\begin{array}{cc}
\partial_{\beta} \partial^{\beta} \psi=-4 \pi G \gamma \rho_{0}, & \partial_{\beta} \partial^{\beta} \mathbf{D}=-\frac{4 \pi G}{c^{2}} \mathbf{J}, \\
\partial_{\beta} \partial^{\beta} \varphi=\frac{\gamma \rho_{0 q}}{\varepsilon_{0}}, & \partial_{\beta} \partial^{\beta} \mathbf{A}=\mu_{0} \mathbf{j}, \\
\partial_{\beta} \partial^{\beta} \vartheta=4 \pi \eta \gamma \rho_{0}, & \partial_{\beta} \partial^{\beta} \mathbf{U}=\frac{4 \pi \eta}{c^{2}} \mathbf{J}, \\
\partial_{\beta} \partial^{\beta} \wp=4 \pi \sigma \gamma \rho_{0}, & \partial_{\beta} \partial^{\beta} \mathbf{\Pi}=\frac{4 \pi \sigma}{c^{2}} \mathbf{J} .
\end{array}
$$

The inhomogeneous wave equations (36) for the field tensors are also simplified, because the terms with curvature vanish. Since the tensor components are expressed in terms of the fields' strength vectors and the fields' solenoidal vectors, the wave equations can be written for each such vector:

$$
\begin{array}{cc}
\partial_{\beta} \partial^{\beta} \mathbf{E}=-\frac{1}{\varepsilon_{0}} \nabla\left(\gamma \rho_{0 q}\right)-\mu_{0} \frac{\partial \mathbf{j}}{\partial t}, & \partial_{\beta} \partial^{\beta} \mathbf{B}=\mu_{0} \nabla \times \mathbf{j}, \\
\partial_{\beta} \partial^{\beta} \mathbf{\Gamma}=4 \pi G \nabla\left(\gamma \rho_{0}\right)+\frac{4 \pi G}{c^{2}} \frac{\partial \mathbf{J}}{\partial t}, & \partial_{\beta} \partial^{\beta} \mathbf{\Omega}=-\frac{4 \pi G}{c^{2}} \nabla \times \mathbf{J}, \\
\partial_{\beta} \partial^{\beta} \mathbf{S}=-4 \pi \eta \nabla\left(\gamma \rho_{0}\right)-\frac{4 \pi \eta}{c^{2}} \frac{\partial \mathbf{J}}{\partial t}, & \partial_{\beta} \partial^{\beta} \mathbf{N}=\frac{4 \pi \eta}{c^{2}} \nabla \times \mathbf{J}, \\
\partial_{\beta} \partial^{\beta} \mathbf{C}=-4 \pi \sigma \nabla\left(\gamma \rho_{0}\right)-\frac{4 \pi \sigma}{c^{2}} \frac{\partial \mathbf{J}}{\partial t}, & \partial_{\beta} \partial^{\beta} \mathbf{I}=\frac{4 \pi \sigma}{c^{2}} \nabla \times \mathbf{J} .
\end{array}
$$

It follows from (38) and (39) that in the empty space, where there are no charges and currents and therefore $\rho_{0 q}=0, \mathbf{j}=0$, the following relations must hold for the field potentials, the electric field strength and the magnetic field of the electromagnetic wave: $\partial_{\beta} \partial^{\beta} \varphi=0, \partial_{\beta} \partial^{\beta} \mathbf{A}=0, \partial_{\beta} \partial^{\beta} \mathbf{E}=0$, $\partial_{\beta} \partial^{\beta} \mathbf{B}=0$. The similar relations in the empty space, in the absence of matter and its fluxes, must also be valid for the gravitational field in the framework of the covariant theory of gravitation: $\partial_{\beta} \partial^{\beta} \psi=0, \partial_{\beta} \partial^{\beta} \mathbf{D}=0, \partial_{\beta} \partial^{\beta} \boldsymbol{\Gamma}=0, \partial_{\beta} \partial^{\beta} \boldsymbol{\Omega}=0$. 
Although equations (37) are of the first order, they are vector equations and for each field they contain four separate coupled equations for the corresponding strengths and solenoidal vectors, which makes it difficult to solve them. In contrast, the solutions of equations (38) for the potentials have a standard form and are called solutions with retarded potentials. As a result, we can substitute the potentials found from (38) into the equations of rectilinear motion of the solid body (28) and (29), and we can determine the Lorentz factor $\gamma$ and the velocity $\mathbf{v}$ as functions of the time and coordinates in the Minkowski spacetime. In practice, however, it usually happens in a different way: first, using the potentials found, with the help of (30) the fields' strengths and solenoidal vectors are calculated, and then they are substituted into the equations of rectilinear motion of the solid body (20), (21), and (22).

Since the components of the field tensors become known, they can be used to calculate the stressenergy tensors of the fields in (3).

\section{Conclusion}

In Section (2) we presented equation (2), which follows from the equation for the metric and is expressed in terms of the stress-energy tensors of the gravitational and electromagnetic fields, the acceleration field and the pressure field. Inside the matter, equation (2) is equivalent to the equation of motion of the matter, written in the form of (5), (7), (13), (14). This becomes possible due to the fact that the fields' energy fluxes transfer the fields' momenta, which are transmitted to the matter's particles during the interaction.

However, the meaning of equation (2) is much broader and it does not reduce only to the equation of motion. Indeed, equation (2) is valid for the space outside the matter's limits, defining there the balance of the fields' energy in accordance with the generalized Poynting theorem, as well as the corresponding field strengths in the space created by the energy fluxes. Moreover, equation (2) in the limit of the weak field and low velocities, where the effects of spacetime curvature can be neglected, can be integrated over the four-volume. The subsequent application of the divergence theorem reduces integration over the four-volume to integration of the time components of the fields' total stress-energy tensor over the three-volume, if the instantaneous situation is considered at a certain given time point. This leads to the determination of the integral vector, which is conserved in closed systems. In contrast, the equation of motion of the matter in the form of (5) cannot be integrated over the volume and does not result in the integral vector.

The equations of motion are simplified for the ideal solid body that does not rotate in its proper center-of-momentum frame and moves rectilinearly, parallel to itself. In order to describe such a motion, we can apply equations (17) and (18), where the field tensors are used, and equations (19) with the fields' strengths and solenoidal vectors. In the flat Minkowski spacetime, the equations of motion are even more simplified and can be represented in the form of equations (20), (21) and (22), which show the rate of change of the energy density and the momentum density, respectively, depending on the value of the strengths and solenoidal vectors inside the matter.

In Section 4, the equation of motion in the form of (24-27) was fully expressed in terms of the derivatives of the fields' scalar and vector potentials taken with respect to time and coordinates. For the rectilinear motion of the solid body in the flat Minkowski spacetime, the equation of motion can be written in the form of two equations (28) and (29), equivalent to equations (20) and (21).

The fact that equations of motion (26) and (27) coincide with equations (14) can be proved using the definitions of the strengths and solenoidal vectors in terms of the fields' scalar and vector potentials indicated in (30). We provided this proof in Section 5.

Due to the relationship between the strengths and solenoidal vectors on the one hand, and the fields' scalar and vector potentials on the other hand, field equations (32) for the strengths and solenoidal vectors can be transformed into wave equations (35) for the fields' scalar and vector potentials. For the field tensors, we also obtained wave equations (36) in the curved spacetime. For all these equations, we provided their expressions in the framework of the special theory of relativity. 
Thus, the mutual complementarity of the field strengths and field potentials is manifested not only in case of their presence in the Lagrange function and their participation in the equation of motion, but also in the corresponding equations for the fields themselves.

If we know the dependences of the mass density $\rho_{0}$ and the charge density $\rho_{0 q}$, as well as the four-velocity $u^{\mu}$ of the matter's motion at each point of the system at any time, this is enough to determine all the characteristics of the system. Indeed, first we find the mass four-current $J^{\mu}=\rho_{0} u^{\mu}$ and the charge four-current $j^{\mu}=\rho_{0 q} u^{\mu}$, and then, using the equations of the corresponding fields, we calculate the field tensors, as well as the four-potentials of the fields. After that, we can find the stressenergy tensors of the fields, the system's metric, the densities of the four-forces, we can solve the equation of motion and also calculate the energy, momentum, angular momentum, and the integral vector of the system's fields, which is associated with the fields' energy fluxes and is found by integrating equation (2) over the four-volume. In addition, we can also determine the fouracceleration of the matter as the derivative of the four-velocity with respect to the proper time in the curved spacetime: $a^{\mu}=\frac{D u^{\mu}}{D \tau}$.

The solution of inverse problems, in which, for example, the dependence of the four-velocity of the matter is unknown, but the fields' distribution as well as the distribution of mass and charge are specified, can be more difficult. In this case, first it is necessary to reestablish the dependence of the four-velocity of the matter with the help of the field equations, and then to determine the remaining characteristics of the system, including the four-acceleration.

\section{Appendix A. Derivation of the covariant wave equation for the electromagnetic field tensor}

The covariant equations of the electromagnetic field for the electromagnetic tensor $F_{\mu \nu}$ have the following form:

$$
\nabla_{\beta} F^{\alpha \beta}=-\mu_{0} j^{\alpha}, \quad \nabla_{\sigma} F_{\mu v}+\nabla_{v} F_{\sigma \mu}+\nabla_{\mu} F_{v \sigma}=0
$$

where $\mu_{0}$ is the magnetic constant, $j^{\alpha}$ is the charge four-current.

Let us take in (A1) the second equation without the field sources, and apply the covariant derivative $\nabla^{\sigma}$ to it:

$$
\nabla^{\sigma} \nabla_{\sigma} F_{\mu \nu}=-\nabla^{\sigma} \nabla_{v} F_{\sigma \mu}-\nabla^{\sigma} \nabla_{\mu} F_{v \sigma}
$$

Next, we will use the rule for the difference of the second-order covariant derivatives:

$$
\left(\nabla_{\alpha} \nabla_{\beta}-\nabla_{\beta} \nabla_{\alpha}\right) F_{\mu v}=R_{\mu, \alpha \beta}^{\rho} F_{\rho v}+R_{v, \alpha \beta}^{\rho} F_{\mu \rho}
$$

where $R_{\mu, \alpha \beta}^{\rho}$ is the curvature tensor.

In view of this rule, we find:

$$
\begin{aligned}
& -\nabla^{\sigma} \nabla_{v} F_{\sigma \mu}=-g^{\sigma \eta} \nabla_{\eta} \nabla_{\nu} F_{\sigma \mu}=-\nabla_{\nu} \nabla_{\eta} F_{\mu}^{\eta}-g^{\sigma \eta} R_{\sigma, \eta \nu}^{\rho} F_{\rho \mu}-g^{\sigma \eta} R_{\mu, \eta \nu}^{\rho} F_{\sigma \rho} . \\
& -\nabla^{\sigma} \nabla_{\mu} F_{v \sigma}=-g^{\sigma \eta} \nabla_{\eta} \nabla_{\mu} F_{v \sigma}=-\nabla_{\mu} \nabla_{\eta} F_{v}^{\eta}-g^{\sigma \eta} R_{v, \eta \mu}^{\rho} F_{\rho \sigma}-g^{\sigma \eta} R_{\sigma, \eta \mu}^{\rho} F_{v \rho} .
\end{aligned}
$$


Now we will take into account the first equation of the electromagnetic field from (A1) and the antisymmetry of the electromagnetic tensor:

$$
\nabla_{\eta} F_{\mu}^{\eta}=-\nabla_{\eta} F_{\mu}{ }^{\eta}=\mu_{0} j_{\mu}, \quad \nabla_{\eta} F_{v}{ }^{\eta}=-\mu_{0} j_{v}
$$

Consequently, in (A3) and (A4) we will have the following:

$$
-\nabla_{v} \nabla_{\eta} F_{\mu}^{\eta}=-\mu_{0} \nabla_{v} j_{\mu}, \quad-\nabla_{\mu} \nabla_{\eta} F_{v}{ }^{\eta}=\mu_{0} \nabla_{\mu} j_{v}
$$

For the curvature tensor, there is a rule:

$$
g_{\sigma \nu} g^{\rho \mu} R_{\mu, \alpha \beta}^{\sigma}=-R_{v, \alpha \beta}^{\rho} .
$$

After multiplying this equality by $g^{v \eta}$ we obtain the following:

$$
g^{\nu \eta} g_{\sigma \nu} g^{\rho \mu} R_{\mu, \alpha \beta}^{\sigma}=\delta_{\sigma}^{\eta} g^{\rho \mu} R_{\mu, \alpha \beta}^{\sigma}=g^{\rho \mu} R_{\mu, \alpha \beta}^{\eta}=-g^{\nu \eta} R_{v, \alpha \beta}^{\rho} .
$$

Here if we assume that index $\rho=\alpha$, then the following relation will be valid:

$$
g^{\alpha \mu} R_{\mu, \alpha \beta}^{\eta}=-g^{v \eta} R_{v, \alpha \beta}^{\alpha}=-g^{v \eta} R_{v \beta}=-R_{\beta}^{\eta} .
$$

With this in mind, the second term on the right-hand side of (A3) and the third term on the right-hand side of (A4) are transformed as follows:

$$
-g^{\sigma \eta} R_{\sigma, \eta v}^{\rho} F_{\rho \mu}=R_{v}^{\rho} F_{\rho \mu}=-F_{\mu \rho} R_{v}^{\rho}, \quad-g^{\sigma \eta} R_{\sigma, \eta \mu}^{\rho} F_{v \rho}=F_{v \rho} R_{\mu}^{\rho} .
$$

Let us transform the third term on the right-hand side of (A3) and the second term on the right-hand side of (A4), in the latter case we will apply the operation of permutation of the indices $\lambda$ and $\eta$ :

$$
\begin{aligned}
& -g^{\sigma \eta} R_{\mu, \eta v}^{\rho} F_{\sigma \rho}=-R_{\mu, \eta v}^{\rho} F_{\rho}^{\eta}=-g_{\rho \lambda} R_{\mu, \eta v}^{\rho} F^{\eta \lambda}=-R_{\mu \lambda, \eta v} F^{\eta \lambda}, \\
& -g^{\sigma \eta} R_{v, \eta \mu}^{\rho} F_{\rho \sigma}=-R_{v, \eta \mu}^{\rho} F_{\rho}^{\eta}=-g_{\rho \lambda} R_{v, \eta \mu}^{\rho} F^{\lambda \eta}=-R_{v \lambda, \eta \mu} F^{\lambda \eta}= \\
& =-R_{v \eta, \lambda \mu} F^{\eta \lambda}=-R_{\lambda \mu, v \eta} F^{\eta \lambda}=-R_{\mu \lambda, \eta v} F^{\eta \lambda} .
\end{aligned}
$$

In view of (A5), (A7) and (A8), for the sum of (A3) and (A4) in (A2) we find the following:

$$
\nabla^{\sigma} \nabla_{\sigma} F_{\mu v}=\mu_{0} \nabla_{\mu} j_{v}-\mu_{0} \nabla_{v} j_{\mu}+F_{v \rho} R_{\mu}^{\rho}-F_{\mu \rho} R_{v}^{\rho}-2 R_{\mu \lambda, \eta v} F^{\eta \lambda}
$$

The last term on the right-hand side of (A9) can also be transformed:

$$
\begin{aligned}
& -2 R_{\mu \lambda, \eta v} F^{\eta \lambda}=-R_{\mu \lambda, \eta \nu} F^{\eta \lambda}-R_{\mu \lambda, \eta v} F^{\eta \lambda}=-R_{\mu \lambda, \eta v} F^{\eta \lambda}+R_{\mu \eta, \nu \lambda} F^{\eta \lambda}+R_{\mu \nu, \lambda \eta} F^{\eta \lambda}= \\
& =-R_{\mu \eta, \lambda v} F^{\lambda \eta}+R_{\mu \eta, v \lambda} F^{\eta \lambda}+R_{\mu \nu, \lambda \eta} F^{\eta \lambda}=-R_{\mu \eta, v \lambda} F^{\eta \lambda}+R_{\mu \eta, \nu \lambda} F^{\eta \lambda}+R_{\mu \nu, \lambda \eta} F^{\eta \lambda}=R_{\mu v, \lambda \eta} F^{\eta \lambda} .
\end{aligned}
$$

In this case for the curvature tensor we applied the rule 


$$
R_{\mu \lambda, \eta v}+R_{\mu \eta, v \lambda}+R_{\mu v, \lambda \eta}=0
$$

used the operation of permutation of the indices $\lambda$ and $\eta$ in one of the tensor products, and took into account the antisymmetry of the electromagnetic tensor $F^{\lambda \eta}=-F^{\eta \lambda}$, as well as the antisymmetry of the curvature tensor in case of permutation of the adjacent indices in each pair of indices.

Finally, we obtain the following:

$$
\nabla^{\sigma} \nabla_{\sigma} F_{\mu v}=\mu_{0} \nabla_{\mu} j_{v}-\mu_{0} \nabla_{v} j_{\mu}+F_{v \rho} R_{\mu}^{\rho}-F_{\mu \rho} R_{v}^{\rho}+R_{\mu v, \lambda \eta} F^{\eta \lambda}
$$

In (A10), the scalar operator $\nabla^{\sigma} \nabla_{\sigma}$ represents the four-dimensional d'Alembertian in the curved spacetime.

\section{References}

1. Landau L.D. and Lifshitz E.M. Mechanics, 3-rd edition. Pergamon, Oxford (1974).

2. José J.V. and Saletan E.J. Classical dynamics: a contemporary approach. Cambridge University Press (1998).

3. Goldstein H. Classical mechanics, 2-nd edition. Addison-Wesley, Reading (1981).

4. Yousaf Z. Spherical relativistic vacuum core models in a $\Lambda$-dominated era. Eur. Phys. J. Plus, Vol. 132, 71 (2017). doi:10.1140/epjp/i2017-11336-9.

5. Yousaf Z. Stellar filaments with Minkowskian core in the Einstein- $\Lambda$ gravity. Eur. Phys. J. Plus, Vol. 132, 276 (2017). doi:10.1140/epjp/i2017-11547-0.

6. Yousaf Z. Structure scalars of spherically symmetric dissipative fluids with $\mathrm{f}(\mathrm{G}, \mathrm{T})$ gravity. Astrophys. Space Sci., Vol. 363, 226 (2018). doi:10.1007/s 10509-018-3450-7.

7. Fedosin S.G. Estimation of the physical parameters of planets and stars in the gravitational equilibrium model. Canadian Journal of Physics, Vol. 94, No. 4, pp. 370-379 (2016). doi:10.1139/cjp-2015-0593.

8. Fedosin S.G. Two components of the macroscopic general field. Reports in Advances of Physical Sciences, Vol. 1, No. 2, 1750002, 9 pages (2017). doi:10.1142/S2424942417500025.

9. Fedosin S.G. The virial theorem and the kinetic energy of particles of a macroscopic system in the general field concept. Continuum Mechanics and Thermodynamics, Vol. 29, Issue 2, pp. 361-371 (2016). doi:10.1007/s00161-016-0536-8.

10. Fedosin S. The physical theories and infinite hierarchical nesting of matter, Volume 2, LAP LAMBERT Academic Publishing, pages: 420, ISBN-13: 978-3-659-71511-2. (2015).

11. Fedosin S.G. The procedure of finding the stress-energy tensor and vector field equations of any form. Advanced Studies in Theoretical Physics, Vol. 8, pp. 771-779 (2014). doi:10.12988/astp.2014.47101.

12. Fedosin S.G. Four-Dimensional Equation of Motion for Viscous Compressible and Charged Fluid with Regard to the Acceleration Field, Pressure Field and Dissipation Field. International Journal of Thermodynamics, Vol. 18, No. 1, pp. 13-24, (2015). doi:10.5541/ijot.5000034003.

13. Fedosin S.G. The Concept of the General Force Vector Field. OALib Journal, Vol. 3, pp. 1-15 (2016), e2459. doi:10.4236/oalib.1102459. 
14. Fedosin S.G. About the cosmological constant, acceleration field, pressure field and energy. Jordan Journal of Physics, Vol. 9, No. 1, pp. 1-30 (2016\} . doi:10.5281/zenodo.889304.

15. Fedosin S.G. The Principle of Least Action in Covariant Theory of Gravitation. Hadronic Journal, Vol. 35, No. 1, pp. 35-70 (2012). doi:10.5281/zenodo.889804.

16. Fock V. A. The Theory of Space, Time and Gravitation. Pergamon Press, London. (1959). 